\title{
Criticality of the magnon-bound-state hierarchy for the quantum Ising chain with the long-range interactions
}

\author{
Yoshihiro Nishiyama \\ Department of Physics, Faculty of Science, Okayama University, Okayama 700-8530, Japan
}

Received: date / Revised version: date

\begin{abstract}
The quantum Ising chain with the interaction decaying as a power law $1 / r^{1+\sigma}$ of the distance between spins $r$ was investigated numerically. A particular attention was paid to the low-energy spectrum, namely, the single-magnon and two-magnon-bound-state masses, $m_{1,2}$, respectively, in the ordered phase. It is anticipated that for each $\sigma$, the scaled bound-state mass $m_{2} / m_{1}$ should take a universal constant (critical amplitude ratio) in the vicinity of the critical point. In this paper, we calculated the amplitude ratio $m_{2} / m_{1}$ with the exact diagonalization method, which yields the spectral information such as $m_{1,2}$ directly. As a result, we found that the scaled mass $m_{2} / m_{1}$ exhibits a non-monotonic dependence on $\sigma$; that is, the bound state is stabilized by an intermediate value of $\sigma$. Such a feature is accordant with a recent observation based on the non-perturbative-renormalization-group method.
\end{abstract}

PACS. 75.10.Jm Quantized spin models - 05.70.Jk Critical point phenomena - 75.40.Mg Numerical simulation studies $-05.50 .+\mathrm{q}$ Lattice theory and statistics (Ising, Potts, etc.)

\section{Introduction}

The $\mathrm{O}(N)$-symmetric classical spin model with the longrange interactions has been investigated both theoretically $[1,2,3, ?, 5,6,7,8, ?, 10,11,12,13,14,15,16,17$, and experimentally $18,19,20,21,22,23,24,25,26,27,28$. A notable feature is that the power of the algebraic decay affects

Send offprint requests to: the criticality of the order-disorder phase transition 1, 2]. Meanwhile, an extention to the quantum-mechanical version was made $29,30,31,32,33,34$. As for the quantum Ising chain with the long-range interactions decaying as a power law $1 / r^{1+\sigma}$ of the distance between spins $r$, there should appear three distinctive types of criticalities 31]; see Fig. 1] For $\sigma<2 / 3$, the criticality belongs to the mean-field type; namely, the singularity is identical to that 
of the four dimensional $(D=4)$ short-range classical Ising model. On the contrary, in $\sigma>1.75$, the long-range interaction becomes irrelevant, and eventually, the criticality reduces to the $D=2$-short-range-classical-Ising universality class. In the intermediate regime $2 / 3<\sigma<1.75$, the singularity depends soothly on $\sigma$. Accordingly, the fractional dimensionality ranges within $2<D<4$ [7, 8, 31. At both boundaries, particularly, at $\sigma=1.75$, there emerge notorious logarithmic corrections [3, 10, 11, 32, and the details as to the end-point singularities are controversial $4,5,6]$. It has to be mentioned that the abovementioned features are not a mere theoretical interest, because such an adjustable algebraic-decay rate $\sigma$ is realized experimentally [19,20,23].

The criticality chart, Fig. 1, differs from that of the classical counterpart [1,2]. The difference comes from the fact that the real-space and imaginary-time directions are anisotropic for the quantum long-range criticality; the anisotr is characterized by the dynamical critical exponent $z$ 31, and accordingly, the set of scaling relations should be remedied. Aiming to elucidate the quantum-mechanical character of this problem, we shed light on the low-lying spectrum. In Fig. 2, we present a schematic drawing for the dispersion relations as to the low-lying elementary excitations in the ordered phase [35]. Here, the symbol $m_{1(2)}$ denotes the single-magnon (two-magnon-bound-state) mass. Above $2 m_{1}$, there extends a continuum, and the series of bound states $m_{3,4, \ldots}$ should be embedded within the continuum 36, 37]. The bound-state hierarchy, namely, the scaled mass $m_{2,3, \ldots} / m_{1}$, displays a universal character in the vicinity of the critical point. As a matter of fact, according to the non-perturbative renormalization group 35, the scaled mass $m_{2} / m_{1}$ exhibits a non-monotonic dependence on $D$ (fractional dimensionality); see Fig. 3. That is, an intermediate value of $D$ stabilizes the bound state. Rather intriguingly, the hierarchy $m_{2,3, \ldots} / m_{1}$ is relevant to the high-energy physics, that is, the gluon-boundstate spectrum (the so-called glueball spectrum) for the gauge field theory 38,39. The universal values $m_{2,3} / m_{1}$ for the Ising model were explored extensively in this context 37 .

In this paper, we investigate the scaled two-magnonbound-state mass gap $m_{2} / m_{1}$ for the spin- $S=1$ Ising chain with the long-range interactions (1). The extended spin $S=1$ permits us to deal with the generalized (quadratic) interactions $\left(D_{s}, \gamma_{2}\right)$, which admit a clear signature for the bound-state mass (such as the plateau in Fig. 6] mentioned afterward) in the finite-size data. We employed the exact diagonalization method, which enables us to calculate the spectral properties such as $m_{1,2}$ directly without resorting to the inverse Laplace transformation; see Appendix B of Ref. [40]. In fairness, it has to be mentioned that recently, the dynamical properties for the long-range systems were studied with the exact diagonalization and the variational-matrix-product methods [41,42,43].

To be specific, the Hamiltonian for the quantum spin$S=1$ Ising chain with the long-range interactions is given by

$$
\mathcal{H}=-\frac{1}{\mathcal{N}} \sum_{i \neq j} J_{i j} S_{i}^{z} S_{j}^{z}+D_{s} \sum_{i}\left(S_{i}^{z}\right)^{2}
$$




$$
-\Gamma \sum_{i}\left(\frac{S_{i}^{+}}{\sqrt{2}}+\frac{\gamma_{2}}{2}\left(S_{i}^{+}\right)^{2}+\text { h.c. }\right)
$$

with the quantum spin- $S=1$ operator $S_{i}^{ \pm, z}$ placed at each one-dimensional lattice point, $i=1,2, \ldots, L$. Here, the periodic boundary condition is imposed. The summation $\sum_{i \neq j}$ runs over all possible $i$ - $j$ pairs, and the symbol $J_{i j}$ denotes the algebraically decaying interaction, $J_{i j}=1 / \sin (\pi|i-j| / L)^{1+\sigma}$, parameterized by $\sigma$. The Kacnormalization factor $\mathcal{N}$ [41,43] is given by $\mathcal{N}=L^{-1} \sum_{i \neq j} \sin (\pi \mid i-$

$j \mid / L)^{-1-\sigma}$. The summation $\sum_{i}$ runs over all spins $i=$ or $1,2, \ldots, L$, and the single-ion anisotropy $D_{s}$ is incorporated. The transverse magnetic field $\Gamma$, together with its quadratic variant $\gamma_{2}$, drives the ferromagnetic state to the disordered phase. These redundant interaction parameters $\left(D_{s}, \gamma_{2}\right)$ are tuned so as to attain a clear indication for the magnon bound state.

The rest of this paper is organized as follows. In the next section, we present the simulation results for the long-range Ising chain (11). The simulation algorithm is explained as well. In the last section, we address the summary and discussions.

\section{Numerical results}

In this section, we present the numerical results for the long-range quantum Ising chain (1). We employed the exact diagonalization method, which enables us to calculate the mass gaps $m_{1,2}$ directly. The numerical diagonalization was performed within the restricted Hilbert space specified by the quantum numbers such as the zeromomentum $(k=0)$ and the spin-inversion $\left(S_{i}^{z} \rightarrow-S_{i}^{z}\right)$ parity index, \pm . In a preliminary survey, we found that the single-magnon mass $m_{1}$ belongs to the sector

$$
m_{1}=E_{1}^{+}-E_{0}^{+}
$$

with the ground-state (first-excited) energy $E_{0}^{+}\left(E_{1}^{+}\right)$with the parity index + . Similarly, the two-magnon-bound-state mass $m_{2}$ is identified as either

$$
m_{2}=E_{3}^{+}-E_{0}^{+},
$$

$$
m_{2}=E_{2}^{-}-E_{0}^{+}
$$

for small- and large- $\sigma$ regimes, respectively; that is, the character of $m_{2}$ changes for respective regimes, as suggested by Fig. 3 [35.

\subsection{Preliminary survey: Scaling behavior for the single-magnon mass $m_{1}$}

As a preliminary survey, in this section, we investigate the scaling behavior for the single-magnon mass $m_{1}$. To this end, we make use of the scaling theory developed in Ref. 31. The interaction parameters are set to $\left(D_{s}, \gamma_{2}\right)=$ $(0,2)$ throughout this section.

In Fig. 4, we present the scaling plot, $\left(\Gamma-\Gamma_{c}\right) L^{1 / \nu_{-}}$ $L^{z} m_{1}$, with the critical point $\Gamma_{c}=0.527$, the reciprocal correlation-length critical exponent $1 / \nu=0.84$, and the dynamical critical exponent $z=0.60$ for the fixed $\sigma=1.2$ and various system sizes, $(+) L=18,(\times) 20$, and $(*)$ 22. Here, the critical point $\Gamma_{c}=0.527$ was extrapolated via the least-squares fit for the $L^{-1}-\Gamma_{c}(L)$ data with $L=$ $18,20,22$, and the approximative critical point $\Gamma_{c}(L)$ was 
determined by the condition $\left.\partial_{\Gamma} m_{1}\right|_{\Gamma=\Gamma_{c}(L)}=0$ for each L. Similarly, the dynamical critical exponent $z=0.60$ was determined via the least-squares fit for $\left(\frac{L+(L+2)}{2}\right)^{-1}$ $z(L, L+2)$ with $L=16,18,20$, and the approximative dynamical critical exponent $z\left(L, L^{\prime}\right)$ is given by

$$
z\left(L, L^{\prime}\right)=-\frac{\left.\ln m_{1}(L)\right|_{\Gamma=\Gamma_{c}(L)}-\left.\ln m_{1}\left(L^{\prime}\right)\right|_{\Gamma=\Gamma_{c}\left(L^{\prime}\right)}}{\ln L-\ln L^{\prime}}
$$

As for the reciprocal correlation-length critical exponent $1 / \nu=0.84$, we made use of the existing value as addressed in Figs. 2 and 3 of Ref. 31 .

From Fig. 4, we notice that the data collapse into a scaling curve satisfactorily. The single-magnon gap $m_{1}$ opens in the ordered phase $\Gamma-\Gamma_{c}<0$. The gap $m_{1}$ sets a fundamental energy scale in the subsequent analyses in Sec. 2.2 and 2.3

Carrying out simular analyses for various values of $\sigma$, we obtained the dynamical critical exponent $z$. The result is presented in Fig. 5. Here, as an indicator for the error, we accept the deviation between the different leastsquares-fit analyses (abscissa scales), namely, the $\left(\frac{L+(L+2)}{2}\right)$ and $\left(\frac{L+(L+2)}{2}\right)^{-2}$-based extrapolation schemes. In the large$\sigma$ side $(D \rightarrow 2)$, the symmetry between the real-space and imaginary-time directions restores, and the dynamical critical exponent reflects the recovery, $z \rightarrow 1$. On the contrary, in the small- $\sigma$ side, these spaces become asymmetric, $z<1$. We stress that our simulation covers these two extreme cases with the interaction parameters fixed to $\left(D_{s}, \gamma_{2}\right)=(0,2)$.

A few remarks are in order. First, the data at both boundaries, $\sigma=2 / 3$ and 1.75 , should suffer from corrections to scaling $3,10,11,32$; particularly, the latter one at $\sigma=1.75$ has arisen controversies [4,5,6] as to the details of the end-point singularity. Because our main concern is the midst regime $\sigma \approx 1$, we do not pursue the details any further. Second, our data appear to obey an approximative formula 31

$$
z=\sigma / 2
$$

Actually, as mentioned above, we obtained $z=0.60$ for $\sigma=1.2 ;$ the result seems to agree with the formula, Eq. (6). The validity of Eq. (6) is not guaranteed for large $\sigma$ 31] nonetheless. Last, the simulation was performed with the interaction parameters fixed to $\left(D_{s}, \gamma_{2}\right)=(0,2)$. In the subsequent sections, we adjust the interaction parameters for the small- and large- $\sigma$ regimes separately in order to attain a clear indication for $m_{2} / m_{1}$.

\subsection{Scaled two-magnon-bound-state mass $m_{2} / m_{1}$ :}

\section{Small- $\sigma$ side}

-In this section, we analyze the scaled two-magnon-boundstate mass $m_{2} / m_{1}$ in the small- $\sigma$ side. Here, the parameters are fixed to $\left(D_{s}, \gamma_{2}\right)=(-0.1,2)$ so as to attain an appreciable plateau for $m_{2} / m_{1}$.

In Fig. 6, we present the scaling plot, $\left(\Gamma-\Gamma_{c}\right) L^{1 / \nu_{-}}$ $m_{2} / m_{1}$, with the critical point $\Gamma_{c}=0.568$ and the reciprocal correlation-length critical exponent $1 / \nu=0.73$ for $\sigma=0.8$ and various system sizes, $(+) L=18,(\times) 20$, and (×) 22 ; here, the critical point $\Gamma_{c}=0.568$ was determined with the same scheme as in Sec. 2.1, and the reciprocal critical exponent $1 / \nu=0.73$ is taken from Figs. 2 and 3 of Ref. 31. We see a plateau with the height $m_{2} / m_{1} \approx 1.9$ 
in the ordered phase $\left(\Gamma-\Gamma_{c}\right) L^{1 / \nu} \approx-2(<0)$. We arrived at $m_{2} / m_{1}=1.895(10)$ via the least-squares fit for $L^{-1}-m_{2} /\left.m_{1}\right|_{\Gamma=\bar{\Gamma}(L)}$ with $L=18,20,22$; here, the location of the plateau (shallow valley) floor $\bar{\Gamma}(L)$ satisfies $\left.\partial_{\Gamma}\left(m_{2} / m_{1}\right)\right|_{\Gamma=\bar{\Gamma}(L)}=0$ for each $L$, and as an indicator for the error, we accept the deviation between the different least-squares-fit analyses (abscissa scales), namely, the $L^{-1}$ - and $L^{-2}$-based extrapolation schemes. Carrying out simular analyses for various values of $\sigma$, we obtained the estimates as indicated by the symbol $(+)$ in Fig. 7

We address a number of remarks. First, for exceedingly large $\sigma$, the plateau width shrinks, and eventually, the plateau disappears. Such a feature suggests that the bound state (belonging to the $(+)$ branch) is no longer supported by the long-range interactions with exceedingly large $\sigma$. Second, the shoulder around $\left(\Gamma-\Gamma_{c}\right) L^{1 / \nu} \approx-2.5$ grows, as the system size $L$ enlarges. Such a feature indicates the stability of the bound state. Last, around the boundary $\sigma=2 / 3$, the simulation data suffer from the systematic errors, as noted in Ref. 32. Because our concern is to survey the bound-state stabilization around $\sigma \approx 1$, we do not pursue the details any further.

\subsection{Scaled two-magnon-bound-state mass $m_{2} / m_{1}$ :}

\section{Large- $\sigma$ side}

In this section, we analyze the scaled two-magnon-boundstate mass $m_{2} / m_{1}$ in the large- $\sigma$ side. Here, the parameters are fixed to $\left(D_{s}, \gamma_{2}\right)=(0.2,0.25)$.

In Fig. 8, we present the scaling plot, $\left(\Gamma-\Gamma_{c}\right) L^{1 / \nu_{-}}$ $m_{2} / m_{1}$, with the critical point $\Gamma_{c}=0.913$ and the recip- rocal correlation-length critical exponent $1 / \nu=0.88$ for $\sigma=1.3$ and various system sizes, $(+) L=18,(\times) 20$, and (*) 22 ; here, the critical point $\Gamma_{c}=0.913$ was determined with the same scheme as in Sec. 2.1, and the exponent $1 / \nu=0.88$ is taken from Figs. 2 and 3 of Ref. 31. The hilltop height $m_{2} / m_{1} \approx 0.85$ indicates the scaled bound-state mass. Via the least-squares fit for $L^{-1}-m_{2} /\left.m_{1}\right|_{\Gamma=\tilde{\Gamma}(L)}$ with $L=18,20,22$, we obtained an estimate $m_{2} / m_{1}=1.879(17)$; here, the hilltop location $\tilde{\Gamma}(L)$ satisfies $\left.\partial_{\Gamma}\left(m_{2} / m_{1}\right)\right|_{\Gamma=\tilde{\Gamma}(L)}=$ 0 for each $L$, and as an indicator for the error, we accept the deviation between the different least-squares-fit analyses (abscissa scales), namely, the $L^{-1}$ - and $L^{-2}$-based extrapolation schemes. Carrying out simular analyses for a variety of $\sigma$, we obtained a series of results as indicated by the symbol $(\times)$ in Fig. 7 .

We address a number of remarks. First, for exceedingly small $\sigma$, the hilltop location $\tilde{\Gamma}$ shifts into the disordered phase, and the branch terminates. Last, the data around the boundary $\sigma=1.75$ should suffer from the systematic errors [3, 10, 11].

\subsection{Comparison with the preceeding results via the $\sigma \leftrightarrow D$ relation [8]}

In this section, we make a comparison with the preceding results such as the fixed- $D=3$ analyses [37,44,45] and the non-perturbative renormalization group for ${ }^{\forall} D$ [35]. In order to establish a relationship between them and ours, we rely on the $\sigma \leftrightarrow D$ relation $[8,31]$

$$
D=2 / \sigma+1
$$


which is validated for small- $\sigma$ (large- $D$ ) regime; more sophisticated formulas 31] do not take such a closed expression.

The overall features of Fig. 7 and 33 [35] resemble each other; actually, the magnon bound state is stabilized by an intermediate value of $\sigma$ and $D$, respectively. Such stabilization of the bound-state mass is captured by neither meanfield theory $(D=4)$ nor free-fermion picture $(D=2)$.

A number of remarks are in order. First, we consider the case $D=3$. This case corresponds to $\sigma=1$ according to the $\sigma \leftrightarrow D$ relation (7). Our result indicates $m_{2} / m_{1}=$ $1.845(10)$ at $\sigma=1$ (along side of the $(\times)$ branch). On the one hand, by means of the Monte Carlo [37], series expansion [4], and exact diagonalization [45] methods, the estimates, $m_{2} / m_{1}=1.83(3), 1.81$, and $1.84(1)$, respectively, were obtained for the fixed- $D=3$ systems. Additionally, the ${ }^{\forall} D$ non-perturbative-renormalization-group method yields $m_{2} / m_{1}=1.82(2)$ at $D=3[35$. Our result appears to be consistent with these preceeding elaborated analyses. According to the super-universality idea, the long- and the short-range models are related with a relation such as Eq. (77) at least for the large- $D$ side [8. Our data suggest that down to $D=3$, the relationship is retained even for the spectral properties such as $m_{2} / m_{1}$. Second, we turn to considering the case, $D \neq 3$. The non-perturbative renormalization group [35] predicts that the minimum point locates around $D=2.7-2.8$, where the scaled mass takes $m_{2} / m_{1}=1.65-1.7$. On the contrary, our result resolves neither appreciable deviation of the minimum point from $D=3(\sigma=1)$ nor notable drop of $m_{2} / m_{1}$ in the small- $D$ side. The discrepancy may be attributed to the breakdown of a naive $\sigma \leftrightarrow D$ correspondence for such small- $D$ regime. Last, we provide a brief overview on the $\sigma \leftrightarrow D$ relation (7). In the course of the studies $7,8,11$, the concept of the $\sigma \leftrightarrow D$ relation has been developed. As for the quantummechanical system, there was reported a refined formula $D=\left(2-\eta_{S R}(D)\right) \frac{1+z(\sigma)}{\sigma}$ [31]. Here, the symbol $\eta_{S R}(D)$ denotes the critical exponent for the short-range model in $D$ dimensions. The explicit expressions for $\eta_{S R}(D)$ and $z(\sigma)$ are unclear. We resort to the approximate relations, $\eta_{S R}=0$ and $z=\sigma / 2$ (6), which are validated in the large- $D$ (small- $\sigma)$ regime [31. As mentioned above, these formulas lead to the closed expression, Eq. (7).

\section{Summary and discussions}

The quantum spin- $S=1$ Ising chain with the long-range interactions (11) was investigated numerically. So far, as for the classical counterpart, a thorough investigation has been made. Aiming to elucidate the quantum nature of this problem, we shed light on its low-energy spectrum, namely, the single-magnon and bound-state masses, $m_{1,2}$, respectively, in the ordered phase. For that purpose, we employed the exact diagonalization method, which enables us to calculate $m_{1,2}$ directly. As a result, we obtained the $\sigma$-dependent scaled bound-state mass, $m_{2} / m_{1}$, for various values of the algebraically-fall-off exponent, $\sigma$.

Thereby, based on the $\sigma \leftrightarrow D$ relation (7), we obtained the result $m_{2} / m_{1}=1.845(10)$ for $D=3$. Our result is to be compared with the preceding results, $m_{2} / m_{1}=$ $1.83(3), 1.81,1.84(1)$, and $1.82(2)$ via the Monte Carlo 
Fig. 1. The quantum Ising chain with the algebraically decaying interactions $\propto 1 /|i-j|^{1+\sigma}$, Eq. (1), exhibits the orderdisorder phase transition. The criticality (universality class) depends on the fall-off exponent $\sigma$, and the singularity is classified into three regimes 31]. For small $\sigma<2 / 3$, the phase transition belongs to the mean-field type, namely, the $D=4$ classical-Ising universality class. For large $\sigma>1.75$, on the contrary, it is identical to that of the $D=2$ Ising model. In the intermediate regime $2 / 3<\sigma<1.75$, the singularity is controlled by $\sigma$ continuously, and correspondingly, the fractional dimensionality changes within $2<D<4$ [8]. At the boundaries, paticularly, at $\sigma=1.75$, there appear notorious logarithmic corrections [3, 10, 11, 32.

[37, series expansion [4], exact diagonalization [45, and non-perturbative-renormalization-group [35] methods, respectively. Hence, it is indicated that down to $D=3$, the validity of super-universality [8] is retained even for the spectral properties such as $m_{2} / m_{1}$. The magnon-boundstate hierarchy is relevant to the glueball spectrum for the gauge field theory 38,39 . It would be intriguing that such high-energy phenomenology is explored [46] for the magnetic materials [36] with finely-tunable [19,20,23] longrange interactions.

\section{References}

1. M. E. Fisher, S.-k. Ma, and B. G. Nickel, Phys. Rev. Lett. 29 (1972) 917.
Fig. 2. A schematic drawing for the low-lying spectrum of the quantum Ising model in the ordered phase is presented. At the zone center $k=0$, there open the single-magnon- and two-magnon-bound-state-mass gaps, $m_{1,2}$, respectively. Above $2 m_{2}$, there extends a continuum, and the magnon-bound-state hierarchy $m_{3,4, \ldots}$ should be embedded within the continuum. The bound state is stabilized around $D \approx 3$, as shown in Fig. 3

2. J. Sak, Phys. Rev. B 8 (1973) 281.

3. E. Luijten and H. W. J. Blöte, Phys. Rev. Lett. 89 (2002) 025703.

4. M. Picco, arXiv:1207.1018.

5. T. Blanchard, M. Picco, and M. A. Rajabpour, Europhys. Lett. 101 (2013) 56003.

6. P. Grassberger, J. Stat. Phys. 153 (2013) 289.

7. G. Gori, M. Michelangeli, N. Defenu, and A. Trombettoni Phys. Rev. E 96 (2017) 012108.

8. M. C. Angelini, G. Parisi, and F. Ricchi-Tersenghi, Phys. Rev. E 89 (2014) 062120. 


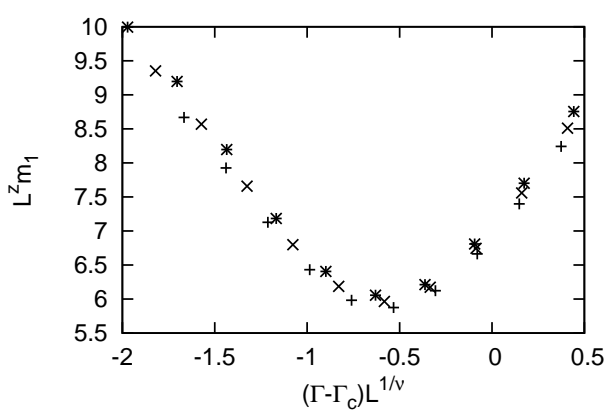

Fig. 3. According to the non-perturbative renormalizationgroup analysis [35, the scaled bound-state mass $m_{2} / m_{1}$ exhibits a non-monotonic dependence on the fractional dimensionality $D$ of the short-range classical Ising model, namely, the $(D-1)$-dimensional quantum Ising model with the shortrange interactions. As a reference, a plot $m_{2} / m_{1}=1.82(2)$ at $D=3$ [35], which is of particular importance, is indicated.

9. J. S. Joyce, Phys. Rev. 146 (1966) 349.

10. E. Brezin, G. Parisi, and F. Ricci-Tersenghi, J. Stat. Phys. 157 (2014) 855.

11. N. Defenu, A. Trombettoni, and A. Codello, Phys Rev. E $92(2015) 052113$.

12. N. Defenu, A. Trombettoni, and S. Ruffo, Phys. Rev. B 94 Fig. 5. The dynamical critical exponent $z$ is presented for var(2016) 224411.

13. R. Goll and P. Kopietz, arXiv:1804.04150. 14. E. Flores-Sola, M. Weigel, R. Kenna, and B. Berche, Eur. $\quad(0,2)$.

Phys. J. Special Topics 226 (2017) 581.

15. T. Horita, H. Suwa, and S. Todo, Phys. Rev. E 95 (2017) 012143.

16. G. Sun, Phys. Rev. A 96 (2017) 043621.

Fig. 4. The scaling plot, $\left(\Gamma-\Gamma_{c}\right) L^{1 / \nu}-L^{z} m_{1}$, with the critical point $\Gamma_{c}=0.527$, the reciprocal correlation-length critical exponent $1 / \nu=0.84$, and the dynamical critical exponent $z=0.60$ is presented for $\sigma=1.2$ and various system sizes, (+) $L=18,(\times) 20$, and $(*) 22$; see text for details. Here, the interaction parameters are set to $\left(D_{s}, \gamma_{2}\right)=(0,2)$.

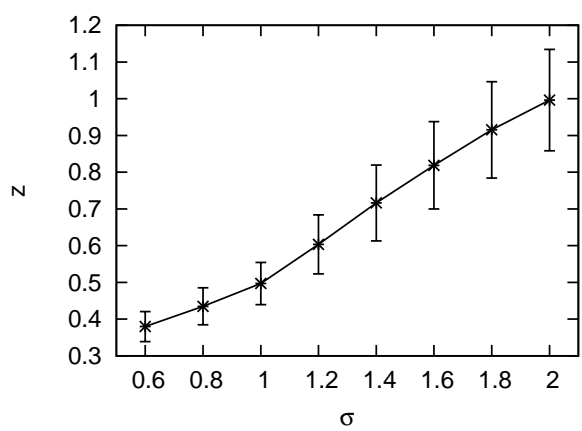
ious values of the algebraically-fall-off exponent $\sigma$; see text for details. Here, the interaction parameters are set to $\left(D_{s}, \gamma_{2}\right)=$ 


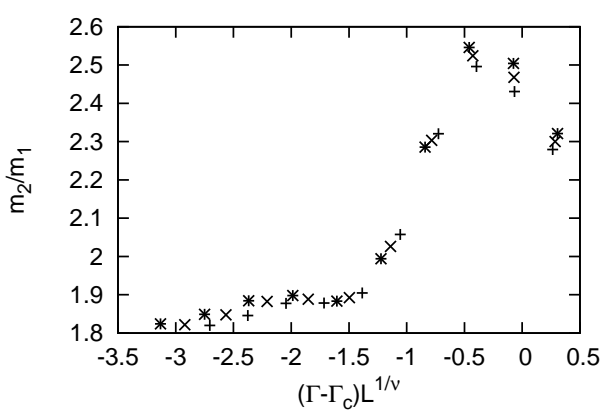

Fig. 6. The scaling plot, $\left(\Gamma-\Gamma_{c}\right) L^{1 / \nu}-m_{2} / m_{1}$, with the critical point $\Gamma_{c}=0.568$ and the reciprocal correlation-length critical exponent $1 / \nu=0.73$ is presented for $\sigma=0.8$ and various system sizes, $(+) L=18,(\times) 20$, and $(*) 22$. Here, the interaction parameters are set to $\left(D_{s}, \gamma_{2}\right)=(-0.1,2)$. The height of the plateau $m_{2} / m_{1} \approx 1.9$ indicates an amplitude ratio; the extrapolated one is plotted in Fig. 7 As the system size enlarges, the shoulder around $\left(\Gamma-\Gamma_{c}\right) L^{1 / \nu} \approx-2.5$ extends leftward, suggesting the stabilization of the bound state in the thermodynamic limit.

17. S. Humeniuk, Phys. Rev. B 93 (2016) 104412.

18. W. Wu, B. Ellman, T. F. Rosenbaum, G. Aeppli, and D.

H. Reich, Phys. Rev. Lett. 67 (1991) 2076.

19. J. W. Britton, B. C. Sawyer, A. C. Keith, C.-C. Joseph Wang, J. K. Freericks, H. Uys, M. J. Biercuk, J. J. Bollinger, Nature 484 (2012) 489.

20. R. Islam, C. Senko, W.C. Cambell, S. Korenblit, J. Smith, A. Lee, E. E. Edwards, C.-C. J. Wang, J. K. Freericks, and C. Monroe, Science 340 (2013) 583.

21. J. G. Bohnet, B. C. Sawyer, J. W. Britton, M. L. Wall, A. M. Rey, M. Foss-Feig, and J. J. Bollinger, Science 352 (2016) 1297.

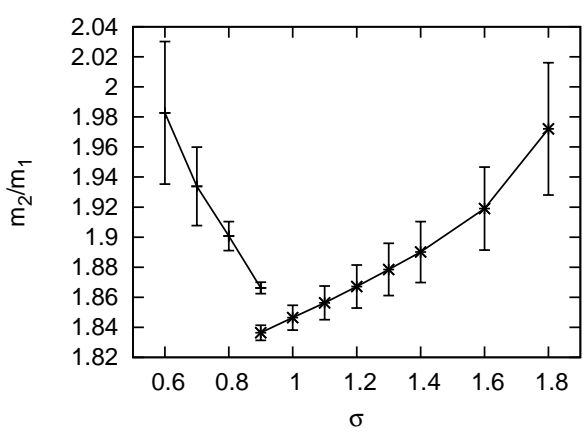

Fig. 7. The scaled bound-state mass $m_{2} / m_{1}$ is presented for various values of the algebraically-fall-off exponent $\sigma$. The plots, $(+)$ and $(\times)$, are determined in Sec. 2.2 and 2.3 , respectively.

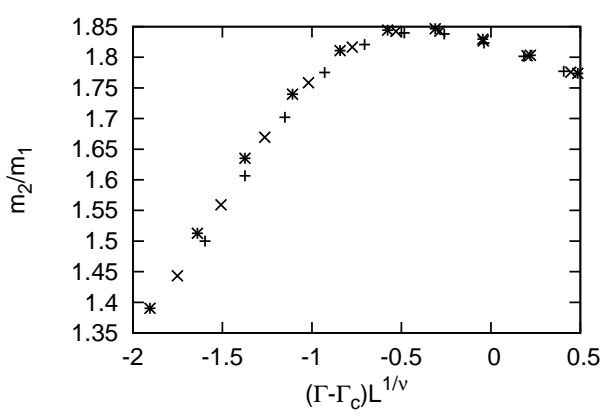

Fig. 8. The scaling plot, $\left(\Gamma-\Gamma_{c}\right) L^{1 / \nu}-m_{2} / m_{1}$, with the critical point $\Gamma_{c}=0.913$ and the reciprocal correlation-length critical exponent $1 / \nu=0.88$ is presented for $\sigma=1.3$ and various system sizes, $(+) L=18,(\times) 20$, and $(*) 22$. Here, the interaction parameters are set to $\left(D_{s}, \gamma_{2}\right)=(0.2,0.25)$. The hilltop height $m_{2} / m_{1} \approx 1.85$ indicates an amplitude ratio; the extrapolated one is plotted in Fig. 7 . 
22. J. Zhang, G. Pagano, P. W. Hess, A. Kyprianidis, P. Becker, H. Kaplan, A. V. Gorshkov, Z.-X. Gong, and C. Monroe, Nature 551 (2017) 601.

23. P. Richerme, Z.-X. Gong, A. Lee, C. Senko, J. Smith, M. Foss-Feig, S. Michalakis, A.V. Gorshkov, and C. Monroe, Nature 511 (2014) 198.

24. P. Jurcevic, B.P. Lanyon, P. Hauke, C. Hempel, P. Zoller, R. Blatt, and C.F. Roos, Nature 511 (2014) 202.

25. A. de Paz, A. Sharma, A. Chotia, E. Maréchal, J.H. Huckans, P. Pedri, L. Santos, O. Gorceix, L. Vernac, and B. Laburthe-Tolra, Phys. Rev. Lett. 111 (2013) 185305.

26. A. Browaeys, D. Barredo, and T. Lahaye, J. Phys. B 49 (2016) 152001.

27. S.A. Moses, J.P. Covey, M.T. Miecnikowski, D.S. Jin, and J. Ye, Nat. Phys. 13 (2017) 13.

28. P. Schauß, M. Cheneau, M. Endres, T. Fukuhara, S. Hild, A. Omran, T. Pohl, C. Gross, S. Kuhr, and I. Bloch, Nature 491 (2012) 87.

29. N. Laflorencie, I. Affleck, and M. Berciu, J. Stat. Mech.: Theory and Experiment, P12001 (2005).

30. A. Dutta and J. K. Bhattacharjee, Phys. Rev. B 64 (2001) 184106.

31. N. Defenu, A. Trombettoni, and S. Ruffo, Phys. Rev. B 96 (2017) 104432.

32. S. Fey and K. P. Schmidt, Phys. Rev. B 94 (2016) 075156.

33. A. W. Sandvik, Phys. Rev. Lett. 104 (2010) 137204.

34. T. Koffel, M. Lewenstein, and L. Tagliacozzo, Phys. Rev. Lett. 109 (2012) 267203.

35. F. Rose, F. Benitez, F. Léonard, and B. Delamotte, Phys. Rev. D 93 (2016) 125018.
36. R. Coldea, D. A. Tennant, E. M. Wheeler, E. Wawrzynska, D. Prabhakaran, M. Telling, K. Habicht, P. Smeibidl, and K. Kiefer, Science 327 (2010) 177.

37. M. Caselle, M. Hasenbusch, P. Provero, Nucl. Phys. B 556 (1999) 575 .

38. V. Agostini, G. Carlino, M. Caselle, and M. Hasenbusch, Nucl. Phys. B 484 (1997) 331.

39. R. Fiore, A. Papa, and P. Provero, Phys. Rev. D 67 (2003) 114508.

40. S. Gazit, D. Podolsky, A. Auerbach, and D. P. Arovas, Phys. Rev. B 88 (2013) 235108.

41. I. Homrighausen, N. O. Abeling, V. Zauner-Stauber, J. C. Halimeh, Phys. Rev. B 96 (2017) 104436.

42. I. Frérot, P Naldesi, T. Roscilde, Phys. Rev. Lett. 120 (2018) 050401.

43. L. Vanderstraeten, M. Van Damme, H. P. Büchler, F. Verstraete, arXiv:1801.00769

44. S. Dusuel, M. Kamfor, K. P. Schmidt, R. Thomale, and J. Vidal, Phys. Rev. B 81 (2010) 064412.

45. Y. Nishiyama, Physica A 413 (2014) 577.

46. B. Svetitsky and L. G. Yaffe, Nucl. Phys. B 210 (1982) 423. 